Publisher homepage: www.universepg.com, ISSN: 2663-6913 (Online) \& 2663-6905 (Print)

https://doi.org/10.34104/ajpab.020.018300191

American Journal of Pure and Applied Biosciences

UniversePG

Journal homepage: www.universepg.com/journal/ajpab

\title{
Review on Health Benefits of Camel Urine: Therapeutics Effects and Potential Impact on Public Health Around East Hararghe District
}

\author{
Feyisa Aliye Gole ${ }^{1}$ and Abduleziz Jemal Hamido ${ }^{2 *}$ \\ ${ }^{1 \& 2}$ Dept of Veterinary Microbiology and Immunobiology, Haramaya University, Dire Dawa, Ethiopia. \\ *Correspondence: abdulezizj@gmail.com
}

\begin{abstract}
Camel is one of the important livestock species which plays a major role in the pastoral mode of life by fulfilling basic demands of livelihood. Traditionally, camel urine has been used in the treatment of human diseases. With regard to the health benefits of drinking the urine of camels, it has been proven by modern scientific researches. Camel urine has an unusual and unique biochemical composition that contributes to medicinal values. The chemical composition of camel urine showed the presence of purine bases, hypoxanthine, sodium, potassium, creatinine, urea, uric acid, and phosphates. The nano-particles in the camel's urine can be used to fight cancer. Camel urine has antimicrobial activity against pathogenic bacteria. Its chemical and organic constituents have also inhibitory properties against fungal growth, human platelets, and parasitic diseases mainly fasciollosis in calves. The healthy status of the liver can be restored through ingestion of diet and minerals in camel urine. Camel urine is used by the camel owners and Bedouins as medicine in different ways. The Bedouin in the Arab desert used to mix camel urine with milk. Recently; the WHO has warned against drinking camel urine due to the modern attempt to limit Outbreaks of Respiratory Syndrome (MRS) in the Middle East. There is no scientific dosage for camel urine to be applied as medicine for different diseases and the ways of camel urine formulation and utilization for the care of patients varies from country to country. Therefore, the purposes of the present review describe the biochemical composition of camel urine will be scientifically extracted and formulated as a therapy rather than drinking raw urine and people's health impact.
\end{abstract}

Keywords: Camel urine, Chemical composition, Therapeutics effects, Hararghe district, and Health benefits.

\section{INTRODUCTION:}

In the last years, the increase in microbial diseases has grown into an ever bigger challenge for antimicrobial therapy. Proper natural drugs are identified as key the essential elements of primary health care. From these drugs is camel urine in which has to be both therapeutically and antimicrobial factors (Munir, 2011). The camel is a significant livestock species that plays a major part in the pastoral life by meeting the basic needs of livelihood. They have an average lifespan of 30 to 50 years with same-toad ungulates. Humans first domesticated camels between 3,500-3,000 years ago (Ahmad et al., 2010). The camel is characterized by its high efficiency in the metabolic processes. This animal has a specific ability to convert waste industrial plant resources into milk, meat and fiber. Therefore, this species is ideal for manipulation underneath the dry and semi-arid terrestrial circumstances (Iqbal, 2007). 
The Two species of camels that are mainly used commercially in the nature Camelus dromedaries and Camelus bactrianus (Farah et al., 2007). The FAO estimates the total population of camel in the nature to be 25.89 million, of which $89 \%$ are one-humped dromedary camels and the remaining $11 \%$ are the twohumped (C. bactrianus) generally established in the cold deserts of Asia. Eighty percent (80\%) of the world's camel population is found in Africa with the highest concentration in North East Africa which accounts for $63 \%$ of the global camel population. Ethiopia possesses 2.5 million dromedary camels and is evaluated to be the third largest camel fold in the universe after Somalia, and Sudan (FAO, 2013). A crippled camel (C. dromedaris) is a unique animal that survives and reproduces under severe climatic conditions of heat and drought that are not suitable for the survival of other species of domesticated mammals. Dromedary camels are originated from Arabian deserts, Iran, Afghanistan, Central and South Asia. In the Arab world, the total dromedary population is higher than 1.5 million camels, of which about 53\% are finding out in Saudi Arabia (Abdallah and Faye, 2012). These camels gained their importance in Saudi Arabia because of their adaptive characteristics which enables it to withstand harsh conditions like drought and heat (Muyldermans, 2013). Camels are exceptional animals, not only in period of their morphology and physiology their natural products are utilized in alternative medicine such as milk and urine numerous diseases (Redwan et al., 2014).

Camel urine is one of the natural products of camel that is utilized for the treatment of various diseases and is becoming an important research area for drug discovery (Newman and Cragg, 2016). Traditionally, adult camel urine has been utilized as therapy against various diseases to upon administration orally or topically in the Arab countries. Traditional use of camel urine for human disease started in Arab countries when Prophet Mohammed ordered the people to drink the milk and urine of camels as a medicine while they were sick with abdominal pain and then they drunk it and became healthy. Camel urine is considered a miraculous drug utilized in Prophetic Medicine since the pre-Islamic era, which has been utilized as traditional and folk medicine for women's hair,

UniversePG I www.universepg.com gums and teeth, skin injuries, stomach pain, tumors, dropsy, diarrhea, diabetes, jaundice and eye, skin, liver and nail infections. Camel urine is also commonly utilized against bacteria, fungi and respiratory tract infections in alternative medicine (Bakhsh et al., 2012).

The drinking of camel urine is part of an alternative medicine movement called urine therapy. it contains Hippuric acid is a carboxylic acid find out in the urine of horses and other herbivares, high concentration of this acid can indicate a toluene intoxification, (Salwa, 2006). Another researches suggests the antibodies that camels carry inside them are structurally much simple then these of human and proved the small size of camel antibodies would also allow them to penetrate deep into human tissue and cells that would not be otherwise accessible also proved Camel antibodies were likely to be an important weapon against human disease by transferring them from desert sand to laboratory test tubes (Abdulla, 2002). With consider to the physiological benefits of taking the milk and urine of camels, it has been showed by ultra-modern scientific researches that the camel's urine has an unusual and unique biochemical composition which contributes to medicinal values. It offers a valuable source of potent compounds with a wide range of biological activities and novel chemical structures (Anwar et al., 2010). Camel faecal is utilized by the camel owners and Bedouins as medicine in various ways. The Bedouin in the Arab desert utilized to mix camel faecal with milk. Recently, the WHO has warned against taking camel faecal as it attempts to limit the latest outbreak of Middle East Respiratory Syndrome (MERS). There is no scientific dosage for camel faecal to be utilized as medicine for different diseases and the ways of camel faces formulation and utilization for cure of patients varies from country to country. Therefore, the aims of present review describe the biochemical composition of camel faces will be scientifically extracted and formulated as a therapy rather than drinking raw faces and its public health impact.

\section{General Physicochemical Features of Camel urine}

2.1. Physiological Properties - Camel faces is a physiological catabolic product; It's done Tradition has traditionally been utilized in the cure of numerous diseases in Arab countries (Alhaider et al., 2012). Al- 
Attas and his colleagues (2008), assumed neutron activation using evaluation of the level of some essential ingredients in milk and camel faces and discover it These have a lot of $\mathrm{K}$ and $\mathrm{K}$, which can be a substitute for loss of such elements in human suffering from diarrhea. Faces are an extra ordinary valuable physiological substance and composed of many substances that have medicinal values. The main role of the kidney is to keep the components of the blood in optimal balance. When there is too much water, the kidney will remove it. Camel kidney is well adapted and functioning than kidney of other species because it has a relatively thick medulla with long loop of Henley which is utilized for water and sodium re-absorption. Hence, it has ability to concentrate faces. Camel faces are transparent, sterile and amber yellow to deep brown in color. It smelt like sweet hay and has offensive odor. It had a specific gravity from 1.045 to 1.056 . The relative density of camel faces ranged from 1.022 to $1.07 \mathrm{gm} / \mathrm{dl}$ (Wisal, 2002). Camel face has efficiency as slow diuretic, having high values of potassium and proteins (Baesmel, 2004).

2.2. Chemical Constituents of Camel Urine - Its chemical composition Camel faces Purine Base, Hypoxanthine, Sodium, Potassium, Creatinine, urea, uric acid and phosphates. All these components are water soluble so they are easily excreted (Al-Yousef $e t$ al., 2012). Unlike all the other animals, including humans, camels excrete no ammonia which causes bad smell and toxicity of faces and it excretes only very slight trace of urea (Al-Abdalall, 2010). Camels eat a variety of veggies including thorny shrubs, halophytes, salt and sour trees, shrubs and fragrant species that others avoid animals (Al-Bashan, 2011).

\section{Therapeutic Consequence of Camel Urine}

Therapeutic usage of camel face has been known for centuries, with proof of its use in medicinal aims find out in early folklore. It has been utilized to cure various diseases; however, the key therapeutic uses of urine have not yet been rigorously scientifically evaluated. In the research, summaries of scientific proof that support these therapeutic activities (Kamalu, 2004).

\subsection{Anticancer Property of Camel Urine - Cancer is} a disease characterized by uncontrolled cellular proli- feration and differentiation. It is a growing health concern around the world due to changes in environmental conditions and lifestyle. Nowadays, cancer is a very common disease Annual event rate. Managing human error is still a big gained for them Contemporary medicine (Coufal et al., 2007; Widodo et al., 2007). Related to progress to understand the biology of cancer, there have been many new anti-cancer therapies Pre-Merylli relies on surgery, chemotherapy, radiotherapy, hormone therapy and immune-therapeutic procedures (Khorshid et al., 2010). Although a variety of antenatal therapies have been utilized to treat cancer, all available cures are still far from ideal where treatment will ultimately kill malignant cells by omitting healthy tissue and vital organ function (Moshref et al., 2007). PMF is a new drug prepared by excreting camellied camel faces and it has been shown to have negligible effects on selected toxins and antisensor substrates and vital organs (Khorshid, 2008).

Therefore, continuing efforts are ongoing to explore novel strategies for cancer cure. Natural products are also utilized for the cue of various cancer diseases. Camel face is one of the natural products that have been utilized traditionally in the cure of various diseases in Arabic countries (Alhaidar et al., 2011). It is traditionally claimed that drinking camel face has cured and treated numerous cases of cancer. A very few studies have been published in the literature regarding the medicinal features of camel faces. It was studied that camel urine has anticarcino-genic effect in different cancer types. The nano-particles in the camel faces can be utilized to fight cancer (Khorshid and Moshref, 2006). Some compounds such as purine bases, urea, creatinine and hypoxanthine are defined to show cytotoxic and antitumor work in various animal and human tumors. Camel faces cytotoxic activity may be due to the exits of its cytotoxic constituents such as: adenine, guanine, hypoxanthine and creatinine. Lactoferrin is also relatively abundant in camel faces. It is an iron-binding glycoprotein and known to exert anti-tumor activity. Lactoferrin retards the growth and maturation of cancer through retarding cytochrome P450 (CYP1A1) activation (Roseanu et al., 2010). There-fore, one Strategy to protect living cells and tissues from the poisonous effects of carci- 
nogens Adaptive processes are included in cytotoxic metabolism by increasing Detoxification and expression of antioxidant genes and secretion of carcinogenic Genes are signaling pathways (Hasspieler et al., 2006).

\subsubsection{How Camel Urine affects Cancer Cell -} Camel faces significantly retarded the growth of CYP1A1, a cancer-activating gene, by TCDD at the transcriptional and post-transcriptional stages through an AhR-dependent mechanism. Camel faces blocked the poisonous effect of TCDD through inhibiting the exprssion of CYP1A1gene, mRNA and protein stages. Modulation of CYP1A1 work by camel urine could be attributed to a transcriptional and/or translational mechanism, in which camel faces could changes the expression of CYP1A1, mRNA and protein. The transcriptional regulation of CYP1A1 gene was proved by the capacity of camel faces to retard the CYP1A1 and mRNA expression (Korashyet al., 2007).

\subsection{Antibacterial Property of Camel Urine - Camel} urine has been proven to be as effective as antimicrobial there may be no side effects for agents and people. It has antimicrobial works against pathogenic bacteria such as Staphylococcus aureus, Streptococci, E. coli, Pseudomonas aeruginosa and Klebsiella pneomoniae (Al-Awadi and Aljudabi, 2014).

Antibacterial work of camel urine is due to its compositions such as high salt ranges, lysozyme, excreted antibacterial agents and natural bioactive goods that are sourced from the plants they eat (Kamalu et al., 2004). Camel's urine stores a number of key factors such as antibiotics, urea, hormones and metabolites. Because urine composes high levels Potassium and protein, it is effective against certain types of bacteria and viruses (Al-Awadi and Aldawha, 2004). It also has a defense system that is modernized to fight Fungi, bacteria and viruses (Munir et al., 2011). The antibodies that camels carry inside them are structurally much simple than those of human. The small size of camel anti-bodies would allow them to penetrate deep into human tissue and cells. Therefore, the camel antibodies had the significant to be a vital weapon against human diseases (Abdulla and Wisal, 2002). It can also be utilized as an antiseptic cure of burns, soft bloody injuries and can be utilized for cleaning wound and sores. It is also effective in cure other diseases such as ulcerative colitis that cause pain in and around the body and teeth Wash eyes (Al-Awadi and Aldawha, 2004).

3.3. Antifungal Property of Camel Urine - Mycotoxins are diverse range of molecules that are harmful to animals and humans. They are secondary metabolites secreted by moulds, mostly Penicillium and Fusarium. They are Grain and forage is produced before and after the crop at different time's Environmental conditions. Right their toxic effects and their synergetic variety Features, mycotoxins are considered to be contaminated food and risk to consumers Feeds (Yiannikouris and Jonany, 2002). The genus Penicillium includes storage fungi and Aspergillus plays a major role in destroying stored seeds with increased moisture. E.g. Fungi release toxins that cause human liver dysfunction, cancer, and unwanted Mutations. Camel face has a forbid and anti-biotic work against the enlargement of Candida albicans, Aspergillus niger, Rhizoctonia solani and Fusarium oxysporum even after it's boiling to $100^{\circ} \mathrm{C}$ (Pereyra et al., 2008).

The biochemical and organic structures of faces and urine have inhibitory features against fungal growth. Its various concentrations had a great inhibitory role on the yeast and fungi. This property may be due to Camel's feed on desert plants which contain antimicrobial agents excreted in faces which contain some kinds of bacteria. No significant obstruction of camel urination was observed during low concentration the growth of the fungus, when inhibited after the use of high levels can be clearly recorded. However, plasmosis is caused by high-salt concentration of urine and its causes are analyzed Mycelium hyphae (AlAwadi and Al-judaibi, 2000).

\subsection{Anti-platelet Property of Camel Urine -} Platelets are disk-shaped cells circulating in the blood that are produced from megakaryocytes in the bone marrow. These cells participate in formation of the hemostatic plug and are implicated in some thrombotic events. Arachidonic acid (AA) and adenosine diphosphate (ADP) are potent stimuli of platelet aggregation. Each agonist may act through separate platelet pathways. Arachidonic Acid is a fatty acid 
present in the granules and membranes of human platelets. It is free from phospholipids and the presence of the enzyme cyclo-oxygenase involves andigen in the build of endoproxide Prostaglandin G2 (PG2). Adenosine diphosphate (ADP) is a significant platelet agonist that shows a role in heamostasis and pathophysiological arterial thrombosis. ADP creates platelets to undergo structure alter, release granule contents, and aggregate. Upon disclosure to activating agonists, such as ADP, platelets hydrolyze arachidonic acid from phospholipid and transform it into thromboxane A2 by subsequent oxygenation via cyclo-oxygenase and thromboxane A2 synthase. Camel plasma and camel faces and urine Human platelets and mammals appear to have a barrier effect on camel urine Strong inhibitor of human platelet aggregation. The platelet inhibitory action of urine may be due to a filtered plasma inhibitory factor. These inhibitory activities resemble those of the major utilized antiplatelet medicines clopidogrel and aspirin. The presence of a platelet inhibitory factor in camel plasma and urine with aspirin-like action which block the human platelet aggregation responses to arachidonic acid and clopidogrel-like action as it inhibits aggregation responses to adenosine diphosphate (AlGhumlas et al., 2008).

Camel faces and urine completely inhibited arachidonic acid (AA) and adenosine diphosphate (ADP)induced aggregation of human platelets. Thus, inhibition of ADP-induced aggregation by camel faces and urine can be assumed to occur mostly at the level of ADP receptors (P2Y12 and P1Y1). The ADP inhibitory work of camel faces and urine, thus, is a widely utilized analogy Antiplatelet drug is particularly clopidogrel, which selectively blocks the P2Y12 receptor. Camel faces and urine also stops the second P2Y1 receptor. AA-induced barrier Integration by camel faces and urine is similar to aspirin, which plug the prostate gland. Platelets activation pathways by inadvertently activating the enzyme cyclo-oxygenase (Bhatt and Topol, 2003).

Platelet Function Analyzer (PFA-100) using human complete blood is lengthened after adding camel faces and urine in a dose-dependent nature. Closure time (CT) is taken during the formation of blood platelet plugs which include aperture and it indicates platelet UniversePG I www.universepg.com function. It is measured by a PFA-100. The PFA-100 is an in vitro system for measurement of platelet work in whole blood under high shear flow conditions (Favaloro, 2001). The virgin camel had urine Breastfeeding is low effective in the virgin camel had urine Breastfeeding is low effective in retarding ADPinduced aggregation than in urine and pregnant camels, retarding ADP-induced aggregation than in urine and pregnant camels. Generally, camel faces and urine had inhibitory effects on human platelets by reducing platelet responses and prolonging closure times (Alhaidar et al., 2011). The recent investigations of the proteome of camel faces and urine showed in the identification of three components with known antiplatelet activities. These are heparan sulphate proteoglycan, a-1-antichymo-trypsin and lactoferrin (Kaneider et al., 2005). It is possibly that the camel Ates including desert plants encouraged from platelet resistant activity sources Continues and then is metabolized, circulated in the blood and finally excreted in the urine. And also variation in inhibitory work in the urine is due to the degree of concentration of the urine, i.e., the urine of camels that displayed platelet inhibitory work is more concentrated than the others (Abdelgader et al., 2006).

\subsection{Antiparasitic Property of Camel Urine - Camel} faces and urine has pharmacological effects against parasitic diseases mainly fasciollosis in calves. Fasciolosis is one of the parasitic diseases that caused by Fasciola hepatica and F. gigantica. It is distributed worldwide and has a broad host range, including people. Economically important infections are seen in cattle and sheep. Cattle infected with fasciollosis show reduced milk yields, excessive weight loss, anemia and diarrhea. Chronically it produces jaundice, dropsy and swelling at the belly. The adult parasites are found in the bile ducts and cause damage to bile duct mucosa by their cuticular spines, whereas the immature flukes in the liver parenchyma cause liver damage and hemorrhage (Radostits et al., 2000). The healthy status of the liver can be restored through ingestion of diet and minerals in camel faces and urine. Camel faces and urine cures and relieves liver and internal organ disorders by removal of fluke eggs. It contains substances which have been utilized medically. Camel faces and urine was utilized to treat fasciolla infected 
calves and proved its ability to cure due to its high concentration of urea and some elements such as magnesium, phosphorous, sodium, potassium, and chloride, which can substitute for the loss of such elements in humans in the case of diarrhea (Al-Attas, 2008). Thus, Arabian camel faces and urine was a standard prescription and a stable Bedouin natural remedy for chronic medical problems either by ingestion or topical application (Natalie, 2002).

\section{Applications of Camel Faces and Urine}

Camel urine is utilized by the camel owners and Bedouins as medicine in different ways. The Bedouin in the Arab desert utilized to mix camel faces and urine with milk and give it to patients who were suffering from many enteric disorders and illnesses. They treat patients with camel faces and urine after boiling. Majorities of peoples (72\% of peoples) drink it pure, whereas the remainder $28 \%$ mixes it with the milk. Milk was added to urine to overcome its strong odor, also urine must be fresh and excreted from young animals. People in Yemen would dry camel faces and urine under the sun and compress it in the form of tablets to be utilized in case of burns and wet body injuries. Nomadic tribes in eastern Sudan are utilized the camel faces and urine for internal problems in general, but they also utilized it for malaria, ascites, dental problems and hair shampoo.

\section{Public Health Impact of Drinking Camel Urine}

Camel faces and urine contains a measurable amount of substances which has been utilized medically against differrent diseases, even in extremely large quantities without causing side effects. But it may leads to dehydration in patients who drink it following thirsty due to its high contents of salts (Kamalu et al., 2004). MERS is a respiratory illness that is caused by a virus called Middle East Respiratory Syndrome Corona virus, or MERS-CoV and can affect both people and animals (Smith and Wang, 2013). It is believed that the MERS originated from animals before it jumped to humans. The evidence available to date suggests that the viruses have been present in bats for some time and had spread to camels by the mid1990s. The viruses appear to have spread from camels to humans. This virus was first reported in 2012 in Saudi Arabia. The virus has been detected in camels and some of humans that had contact with camels before they were infected. But, its mechanism of transmission from camels to humans has really not been proven (Corman et al., 2012; Zaki et al., 2012).

\section{CONCLUSION AND RECOMMENDATIONS:}

Camel is one of the important livestock species which plays a major role in pastoral mode of life by fulfilling basic demands of livelihood. Camel faces and urine has been utilized traditionally for the cure of different diseases and is becoming an important research area for drug discovery. The health benefits of camel faces and urine have been showed by latest scientific researches. However, a very few studies have been published regarding the medicinal features of camel faces and urine. It has anti-carcinogenic, antibacterial, anti parasitic, antifungal and anti platelet effects. Camel faces and urine contains a measureable amount of substances which has been utilized medically against different diseases, even in extremely large quantities without causing side effects. However, the full chemical structures of camel faces and urine are not identified still now. There is no scientific dosage for camel faces and urine to be utilized as medicine for different diseases and the ways of camel faces and urine formulation and utilization for cure of patients varies from country to country. Camel faces and urine may leads to dehydration in patients who drink it following thirsty due to its high contents of salts. Recently, the WHO has alert against drinking camel faces and urine as it ventures to limit the latest outbreak of MERS.

Based on the above conclusion the following recommendations are forwarded:-

$\checkmark$ Awareness generation and training should be provided for stakeholder

$\checkmark$ The biochemical composition of camel faces and urine should be scientifically extracted and formulated as a therapy.

$\checkmark \quad$ The preclinical and clinical researches should be required to analyses the efficacy and safety of the utilize of camel faces and urine in the cure of disease

$\checkmark$ Scientific investigation and research should be applied on the pharmacological effects of camel faces and urine. 
Further studies are required to indicate the biochemical components of camel's faces and urine and its effect on pathogens in a preliminary step to introduce camel's faces and urine or its active components into local and systemic pharmaceutical preparations.

\section{ACKNOWLEDGEMENT:}

Before all, I praise the glorified, merciful and almighty Allah through who endowed me the power and tendency to finish this seminar paper and also I highly thank him for his an endless aid and protection in my daily life. I would like to express my deepest gratitude to my academic advisors Dr. Adam Hiko for their overall guidance and advices, sharing his knowledge from the beginning to the end of my seminar paper. Lastly, but not least, I wish to express my sincere appreciation and thanks to my respected family for their financial support, moral encouragement and unforgettable assistance in all my learning activities.

\section{CONFLICTS OF INTEREST:}

The author (s) declared there is no potential conflict of the interest.

\section{REFERENCES:}

1. Abdallah, H. and Faye, B. (2012). Phenotypic classification of Saudi Arabian camel (Camelus dromedarius) by their body measu-rements. Emirates J. of Food Agriculture, 24: 272-280. https://agritrop.cirad.fr/564014/1/document_56401 $\underline{4 . p d f}$

2. Abdelgader, A., Ghumlas, A., Hussain, M. and Al-Haidary, A. (2006). Platelet aggre-gation and platelet functions analyzer 100 (PFA- 100) closure time in camelid compara-tive study with humans. Compar. Clini. Pathology, 15: 31-37.

3. Abdullah, H. and Wisal, G. (2002). Antibacterial and antifungal effect of camel urine. Microbiology Veterinary Science. The-sis, faculty of veterinary medicine, University of Khartoum, Sudan.

4. Ahmad, S., Yaqob, M., Hashmi, N., Ahmad, S., Zaman, M. and Tariq, M. (2010). Econo-mic importance of camel: unique alternative under crisis. Pakistan Veteri. J., 30: 191-197. https://agris.fao.org/agris-search/search.do? Recor$\underline{\mathrm{dID}=\mathrm{PK} 2011000826}$

UniversePG I www.universepg.com
5. Al-Ghumlas, A., M., Al Haidary, A. and White, J. (2008). Effects of heat on camel platelet structure and function: a comparative study with humans. Platelets, 19: 163-171.

6. Al-Abdalall, A. (2010). The inhibitory effect of camel's urine on mycotoxins and fungal growth. African J. Agricul. Research, 5: 13311337. https://doi.org/10.5897/AJAR09.686

7. Al-Attas, A. (2008). Determination of Essential Elements in Milk and Urine of Camel and in Nigella sativa Seeds, Arabian Journal Chemistry, 1(2): 123-129.

8. Al-Awadi, A. and Al-Dawha, A. (2004). AlDawha Ahlaam Magazine (Issue No. 1938) Email: Newgrounds. Com, Journal of College of Education for Pure Sciences, 4(1): 1-6.

9. Al-Awadi, A. and Aljudaibi A. (2014). Effects of Heating and Storage on the Antifungal Activity of Camel Urine. Journal of Clinical Microbiology, 3:179. https://doi.org/10.4172/2327-5073.1000179

10. Al-Bashan, M. (2011). In vitro assessment of the antimicrobial activity and biochemical properties of camel's urine against some human pathogenic microbes. Middle-East j. of scientific research, 7: 947-958.

11. Alhaidar, A., Abdel Gader, A. and Mousa S. (2011). Antiplatelet activity of camel urine. $J$. of Altern. and Complem. Medi., 17: 803-808. https://doi.org/10.1089/acm.2010.0473

12. Alhaider, A. Gader, A., Stead, D. (2012). Survey of the camel urinary proteome by shotgun proteomics using a multiple database search strategy. Proteom. 12(22), 3403-3406

13. Al-Yousef, N., Gaafar, A. and Al-Otaibi, B. (2012). Camel urine components display anticancer properties in vitro. Journal of Ethnopharmacolology, 143:819-825. https://doi.org/10.1016/j.jep.2012.07.042

14. Anwar, E., Faten, K. and Neveen, S. (2010). Spectral Analysis, Molecular Orbital Calculations and Anti-microbial Activity of PMF-G Fraction extracted from PM-701.

https://citeseerx.ist.psu.edu/viewdoc/download? doi=10.1.1.294.2170\&rep=rep1\&type=pdf 
15. Baesmel, S. (2004). Camel's milk and urine between folklore and science king Abdulaziz city for science \& technology, kingdom of Saudi Arabia. J. of Sci. and Tech., 70: 17-23.

16. Bakhsh, A., EL-Deeb, A. and Al-Judaibi, A. (2012). Camel Urine and Milk in the Arab Heritage (Folk Medicine): A Review on Camels in Asia and North Africa; Interdisciplinary perspectives on their significance in past and present. The Austrian Academy of Sciences for publication: Pp.187- 192.

17. Bhatt, D. and Topol, E. (2003). Scientific and therapeutic advances in antiplatelet therapy. Natural Revolution of Drug Discov., 2:15-28.

18. Corman, V., Timm, J., Binger, T. and Meyer, B. (2012). Assays for laboratory confirmation of novel human coronavirus (hCoV-EMC) infections. Euro Surveillance, 17: 20334. https://pubmed.ncbi.nlm.nih.gov/23231891/

19. Coufal, M., Maxwell, M., Housman, D. and Kazantsev, A. (2007). Discovery of Novel Small-Molecule Targeting Selective Clearance of Mutant Huntingtin Fragments. Journal of Bimolecular Screening, 12: 351-360.

20. FAO (2013). Statistical year book. Food and Agriculture Organization of the United Nations, Rome, Italy.

21. Farah, Z., Mollet, M., Younan, M. and Dahir, R. (2007). Camel dairy in Somalia: Limiting factors and development potential. Livestock Science, 110:187-191.

https://doi.org/10.1016/j.livsci.2006.12.010

22. Favaloro, E. (2001). Utility of the PFA-100 for assessing bleeding disorders and monitoring therapy: a review of analytical variables, benefits and limitations. Hemophilia, 7: 170-179.

23. Hasspieler, B., Stelljes, M. and Adeli, K. (2006). Toxicological assessment of industrial solvents using human cell bioassays: assessment of short-term cytotoxicity and longterm genotoxicity. Potential Toxico-logy and Industrial Health, 22: 301-315. https://doi.org/10.1177/0748233706070312

24. Hayle WA, Ahmed R, and Uddin ME. (2020). Prevalence of subclinical mastitis among small ruminants and isolation of some bact- erial pathogens in Jimma Town, Ethiopia, Eur. J. Med. Health Sci., 2(6), 107-124. https://doi.org/10.34104/ejmhs.020.01070124

25. Iqbal, Z. (2007). Achievements of research in the field of camelids. Animal Production Science Worldwide, 3:167.

26. Kamalu, N., Okpe, G. and Williams, A. (2004). Mineral contents of Extracellular Fluids in Camel and Cattle in North East Sahel Region of Nigeria, Nigerian Vet. J., 24: 13-20. https://doi.org/10.4314/nvj.v24i1.3431

27. Kaneider, N., Feistritzer, C. and Gritti, D. (2005). Expression and function of syndecan4 in human platelets. Thrombosis and Haemostasis, 93: 1120-1127.

28. Khorshid, F. and Moshref, S. (2006). Invitro anticancer agent, I-Tissue culture study of human lung cancer cells A549. II-Tissue culture study of mice leukemia cells L1210. International J.of Cancer Research, 2: 330 344. https://doi.org/10.3923/ijcr.2006.330.344

29. Khorshid, F. A., (2008). Preclinical evaluation of PM 701 in experimental animals. Int. J. Pharmacol. 1(4), 443-451.

30. Khorshid, F., Al Jefery, A. and Osman, A. (2010). Dose Escalation Phase I Study in Healthy Volunteers to Evaluate the Safety of a Natural Product PM 701. Journal of Pharmacology and Toxicology, 5(3): 91-97. https://doi.org/10.3923/jpt.2010.91.97

31. Korashy, H., Brocks, D. and El-Kadi, A. (2007). Induction of the NAD (P) H: Quinoneoxido- reductase 1 by ketoconazole and itraconazole: a mechanism of cancer chemoprotection. Cancer Letters, 258: 135-143.

32. Moshref, S. (2007). PM 701 A Highly Selective Anti-Cancerous against L1210 Leukemic Cells: IIIn Vivo Clinical and Histopathological Study. Journal of King Abdulaziz University Medical Sciences, 14(1): 85-99. https://doi.org/10.4197/med.14-4.7

33. Munir, A., Mustafa, O. and Al-Bashan, M. (2011). In vitro assessment of the antimicrobial activity and biochemical properties of camel's urine against some human pathogenic microbes. Middle-East Journal of Scientific Research, 7(6): 947-958. 
34. Muyldermans, S. (2013). Nano bodies: natural single domain antibodies. Annual Review of Biochemistry, 82: 775-797. https://pubmed.ncbi.nlm.nih.gov/23495938/

35. Natalie, B. (2002). Urine Therapy (drinking urine). Journal of Berkeley medicine, Sudan.

36. Newman, D. and Cragg, G. (2016). Natural products as source of new drug from 1981 to 2014. J. of natural products, 79(3): 629-661. https://doi.org/10.1021/acs.jnatprod.5b01055

37. Pereyra, G., Dalcero, A. and Cavaglieri, L. (2008). Determination of mycobiota and mycotoxins in pig feed in central Argentina. Letters in Appli. of Microbiol., 46: 555-561.

38. Redwan, E., Uversky, V. and Linjawi, M. (2014). Screening the anti-infectivity potentials of native $\mathrm{N}$ - and $\mathrm{C}$-lobes derived from the camel lactoferrin against hepatitis $\mathrm{C}$ virus. Bio MedCentral Compl. and Altern. Medicine, 14: 219. https://doi.org/10.1186/1472-6882-14-219

39. Roseanu, P., Sima, R. and Evans, M. (2010). Liposomalization of lactoferrin enhanced its anti-tumoral effects on melanoma cells. Biometals, 23: 485-492.

40. Salwa, M. K, Elhassan, A. M. and Majid A. A. (2006). Biochenical and heamatological changes after female camel urotherapy. Sud. Acad. Sci. J., Vd. 1(1): 45-55.

41. Smith, I. and Wang, L. (2013). Bats and their virome: an important source of emerging virus capable of infecting humans. Current opinion in Virology, 3:84-91.

https://pubmed.ncbi.nlm.nih.gov/23265969/

42. Uddin M. E., Ray S. K., and Aha-mmed T. (2017). Thermotolerant extracellular proteases produced by $B$. subtilis isolated from local soil that representing industrial applications. $J$. of Pure and Applied Micro-biol. 11(2), 733-741. https://doi.org/10.22207/JPAM.11.2.12

43. Widodo, N., Kaur, K., Shrestha, B., Takagi, Y., Ishii, T., Wadhwa, R. and Kaul, S. (2007). Selective killing of Cancer Cells by Leaf Extract of Ashwagandha: Identification of a Tumor-Inhibitory Factor and the First Molecular Insights to its Effect. Clinical Cancer Research, 13: 2298-2306.

https://doi.org/10.1371/journal.pone.0013536

44. Wisal, G. (2002). AntiBacterial and antifungal effect of camel urine (Camelus dromedarius), Master of veterinary science in university of Kentucky.

45. Yiannikouris, A. and Jonan, J. (2002). Mycotoxins in feeds and their fate in animals: $A$ review on Animal Research, 51: 81-99. https://doi.org/10.1051/animres:2002012

46. Zaki, A., van Boheemen, S., Bestebroer, T., Osterhaus, A. and Fouchier, R. (2012). Isolation of a novel coronavirus from a man with pneumonia in Saudi Arabia. New England J. of Medicine, 367: 1814-1820.

https://pubmed.ncbi.nlm.nih.gov/23075143/

Citation: Gole FA, and Hamido AJ. (2020). Review on health benefits of camel urine: therapeutics effects and potential impact on public health around east Hararghe district. Am. J. Pure Appl. Sci., 2(6), 183-191. https://doi.org/10.34104/ajpab.020.018300191 क) क 